Purdue University

Purdue e-Pubs

2004

\title{
Analysis and Prediction of Constriction Resistance for Contact Between Rough Engineering Surfaces
}

\author{
A. F. Black \\ V. Singhal \\ S V. Garimella \\ Purdue University, sureshg@purdue.edu
}

Follow this and additional works at: http://docs.lib.purdue.edu/coolingpubs

Black, A. F.; Singhal, V.; and Garimella, S V., "Analysis and Prediction of Constriction Resistance for Contact Between Rough Engineering Surfaces" (2004). CTRC Research Publications. Paper 296.

http://docs.lib.purdue.edu/coolingpubs/296

This document has been made available through Purdue e-Pubs, a service of the Purdue University Libraries. Please contact epubs@purdue.edu for additional information. 


\title{
Analysis and Prediction of Constriction Resistance for Contact between Rough Engineering Surfaces ${ }^{\dagger}$
}

\author{
Anthony F. Black, Vishal Singhal and Suresh V. Garimella \\ Cooling Technologies Research Center \\ School of Mechanical Engineering, Purdue University \\ West Lafayette, Indiana 47907-1288
}

\begin{abstract}
The constriction resistance of a semi-infinite cylinder terminating in the frustum of a cone in a gaseous environment is analyzed numerically. The variation of constriction resistance with the ratio of contact radius to cylinder radius, the cone angle, and the gas-to-substrate thermal conductivity ratio is investigated. Nonlinear curve fitting is used to develop a comprehensive predictive correlation for the constriction resistance as a function of these parameters. The parameters are investigated over a wide range of values covering the range expected in practical applications. The correlation could be used in conjunction with an appropriate surface deformation model to predict the thermal contact resistance between rough metallic surfaces.
\end{abstract}

\footnotetext{
${ }^{\dagger}$ Submitted for publication in AIAA Journal of Thermophysics and Heat Transfer, August 2002, and in revised form May 2003.

* Author to whom correspondence should be addressed, Tel.: 765-494-5621, E-mail: sureshg@ecn.purdue.edu
} 


\section{Nomenclature}

$\begin{array}{ll}a & \text { contact spot radius } \\ b & \text { cylinder radius } \\ d / d z & z \text {-gradient } \\ d / d r & r \text {-gradient } \\ F & \text { constriction alleviation factor } \\ k & \text { thermal conductivity }(\mathrm{W} / \mathrm{m}-\mathrm{K}) \\ L & \text { length of cylinder } \\ q & \text { heat flow (W) } \\ R & \text { thermal resistance }(\mathrm{K} / \mathrm{W}) \\ r & \text { radial direction } \\ T & \text { temperature } \\ z & \text { axial direction } \\ \kappa & \text { conductivity ratio in the correlation } \\ q & \text { contact angle or frustum cone angle (rad) }\end{array}$

Subscripts and Superscripts

0 interface plane

* extrapolated

cons constriction

cyl cylinder

half semi-infinite half-space

gas gas gap fluid

sub cylinder (substrate) material 


\section{INTRODUCTION}

Thermal contact resistance is a pervasive problem in the design of microelectronics, avionics, and space thermal management systems [1,2]. Whenever two surfaces come into contact, a resistance to heat flow exists at the interface. This resistance is a result of the small fraction (usually around 1-2\% [3]) of the nominal area that is actually in contact at any given interface, as illustrated in Figure 1. There are three main modes of heat transfer through such interfaces: conduction through the contact spots, conduction through the gas gap, and radiation across the gas gap. Convection in the gas gap is insignificant due to the small length scale of the gas gap [2]. Also, at temperatures below $500 \mathrm{~K}$, radiation does not have a significant effect [4]. Moreover, since the gas gap is usually much less conductive than the substrate material, most of the heat tends to be constrained to flow through the small contact spots. The resistance posed by the constrained heat flow through these contact spots is known as constriction resistance and is characterized by the constriction and subsequent spreading of the heat flow path lines at the interface (Figure 1). The contact resistance is the combined effect of the constriction resistances at all the contact spots at an interface. Hence accurate determination of constriction resistance is necessary to calculate contact resistance with good accuracy. The constriction and spreading causes an additional temperature drop across the interface over that incurred when there is no constriction. Constriction resistance is defined as the additional temperature drop due to constriction divided by the rate of heat flow through the interface:

$$
R_{\text {cons }}=\frac{T^{*}-T_{0}}{q}
$$


In Equation (1), $T^{*}$ would be the temperature at the interface plane if there were no constriction, as shown in Figure 2. An analytical solution [5] for the constriction resistance of a small opening of radius $a$ in a semi-infinite half-space gives:

$$
R_{\text {cons-half }}=\frac{1}{4 k_{\text {sub }} a}
$$

A real interface is characterized by several contact spots of varying radii, which are randomly distributed over the interface. Constriction resistance at an interface is generally modeled by several heat flux columns (cylinders), each feeding an individual contact spot as shown schematically in Figure 3 [2]. However, many different approaches have been used to model the area near the contact plane of the cylinders. Most existing work considers constriction resistance from a semi-infinite cylinder (the heat flux column) terminating, at the contact surface, in a small radius of contact as illustrated in Figure 4 [6]. Mikic and Rohsenow [7] showed that the constriction resistance for this case is proportional to that of the semi-infinite half-space:

$$
R_{\text {cons-cyl }}=F \cdot R_{\text {cons-half }}
$$

in which $F$ is known as the constriction alleviation factor. Since the constriction resistance is alleviated for the semi-infinite cylinder when compared to a semi-infinite half-space, $F$ is less than 1. A number of studies have investigated the constriction alleviation factor for bare contacts [8,9]. Cooper et al. [10] obtained a simple correlation for the constriction alleviation factor for such contacts, given by:

$$
F=\left(1-\frac{a}{b}\right)^{1.5}
$$

However, at a real contact, the constriction resistance will depend on the contact angle, which is the angle between the slope of an asperity and the contact plane, as well as the conductivity of the gas gap [11]. Olsen et al. [4] argued that the constriction resistance should be modeled with a 
semi-infinite cylinder terminating in the frustum of a cone as shown in Figure 5. They analyzed the problem numerically using a finite-difference approach on a structured axisymmetric grid. A dedicated FORTRAN code employing Gauss-Seidel iteration with successive over-relaxation was used to solve the resultant governing equations. Although the methodology used in their work is suited to the current problem, several improvements can be made to increase the accuracy and utility of the results:

1. The model uses a non-graded structured mesh, which is not suited to the large nonuniformities in heat flux that are encountered at a typical constriction.

2. For very small contact radii (small $a / b$ ), the results obtained from the model are subject to greater inaccuracies (and slower convergence rates) because of the large spatial variations in temperature gradient.

3. The results are not in a ready-to-use form since no generalized correlations were provided.

These problems can be overcome by using unstructured grids with a high grid density in the regions of large heat flux non-uniformities, as is done in the present work using a commercial computational fluid dynamics (CFD) program.

Typical configurations of the asperities on rough engineering surfaces were obtained from topographical analyses of a number of ground and bead-blasted flat metallic surfaces. This makes it possible to develop a correlation that is applicable to most practical contacts. The aim of the present work is to generate a comprehensive database of constriction resistance values for a wide range of parameters, and to thereby propose a predictive correlation which relates the constriction resistance to the governing parameters. These governing variables include the ratio of the contact spot radius to the cylinder radius $(a / b)$, the contact angle of the asperity $(\theta)$, and 
the ratio of the conductivity of the gas gap (usually air) to the conductivity of the substrate material $\left(k_{\text {gas }}\left(k_{s u b}\right)\right.$, over a range that is appropriate for real engineering surfaces. This correlation, in conjunction with a surface deformation model as described in Singhal and Garimella [12], can then be used to predict the contact conductance at real interfaces. This surface deformation model provides the real area of contact for each contacting asperity at an interface by considering the surface profiles of the two contacting surfaces, material properties and contact pressure. The constriction resistance through the contacting asperities is then obtained from a constriction resistance model, such as the one developed in the present work. Addition of all the constriction resistances gives the value of contact resistance at the interface. Further details of the surface deformatino model and the method used to compute the contact resistance from constriction resistance are available in [12].

\section{NUMERICAL MODEL AND ANALYSIS}

The constriction encountered in an asperity was modeled using a semi-infinite cylinder of radius $b$, terminating in the frustum of a cone of tip radius $a$. A two-dimensional, axisymmetric model of the asperity as shown in Figure 5 was used for numerical modeling. The numerical analysis was performed for different values of $\theta, a / b$ and $k_{g a s} / k_{s u b}$. In the computations, the contact angle was varied from 0.0175 to 0.628 radians in approximately 0.035 -radian increments ( 1 to 36 deg in roughly 2 -deg increments), while $a / b$ was varied from 0.01 to 0.1 in increments of 0.01; these were determined to be the appropriate ranges based on surface topography analysis data [12] for several different metals that had been ground and bead-blasted to obtain centerline average surface roughnesses ranging from 1 to $15 \mu \mathrm{m}$. The conductivity ratio $\left(k_{\text {gas }} / k_{\text {sub }}\right)$ was

varied from $5.83 \times 10^{-5}$ to $1.61 \times 10^{-3}$ by using a constant gas conductivity (that of air, $k_{\text {gas }}=$ 
$0.0242 \mathrm{~W} / \mathrm{mK}$ ) and varying the conductivity of the substrate from 415 (silver) to 15 (stainless steel) W/mK. The grid-generation package GAMBIT [13] was used to generate unstructured grids for 160 different cylinder geometries. Each grid has approximately 3000 elements with a very fine resolution in the area of the contact spot and the frustum of the cone. Mesh independence was determined by more than tripling the number of mesh elements to 11000 . This resulted in a change in heat flow through the top of the domain of less than $1 \%$ from the case of the coarser grid.

The boundary conditions used on the domain are as follows. An axisymmetric boundary condition was used for the central axis of the domain ( $r=0$ in Figure 5):

$$
\frac{\partial T}{\partial r}(0, z)=0
$$

It was assumed that there is no thermal interaction between adjacent heat flow cylinders (Figure 3). Therefore, an adiabatic condition was imposed on the outside surface of the cylinder $(r=b$ in Figure 5):

$$
\frac{\partial T}{\partial r}(b, z)=0
$$

Uniform temperature boundary conditions were used for the top and bottom ends of the domain:

$$
\begin{aligned}
& T(r, L)=125^{\circ} \mathrm{C} \\
& T(r, 0)=25^{\circ} \mathrm{C}
\end{aligned}
$$

The semi-infinite condition for a cylinder can be approximated if the cylinder is long enough so that the heat flow is uniform across its top boundary. Olsen et al. [4] have shown this to be the case if the length of the cylinder is four times its radius. A plot of the heat flux at the top of the cylinder for the most restrictive case of $a / b=0.01, \theta=0.628$-radian, and $k_{\text {gas }} / k_{\text {sub }}=$ $1.61 \times 10^{-3}$, is shown in Figure 6. This shows that the heat flow is uniform at the upper end of 
the cylinder, and should be even more so for all other cases. A two-sided cylinder (mirrorimaged across $z=0$ ) as shown in Figure 7 was also modeled to determine the validity of the constant temperature condition at the interface. For identical temperature drops across each cylinder of $100^{\circ} \mathrm{C}$ (constant temperatures of $125^{\circ} \mathrm{C}$ at the top and $-75^{\circ} \mathrm{C}$ at the bottom of the domain), the interface temperature was found to be fairly constant even in this worst-case example as seen from Figure 8. This proves the validity of using a constant temperature condition over the interface.

The commercially available CFD software FLUENT [14] was used to numerically model and solve the problem using the boundary conditions discussed above. The conservative convergence criterion used resulted in a heat flow mismatch through the bottom $(z=0)$ and the top $(z=L)$ of the domain of less than $2 \%$ for all cases. The heat flow through the domain is computed from the energy equation and is a standard output parameter in FLUENT. The heat flow in conjunction with Fourier's law was used to compute the temperature gradient $(d T / d z)$ at the top of the domain. The heat flow and temperature gradient were used to calculate the constriction resistance as:

$$
R_{\text {cons }-c y l}=\frac{T_{z=L}+L\left[\frac{d T}{d z}\right]_{z=L}-T_{z=0}}{q}
$$

The constriction alleviation factor $F$ was obtained by dividing Equation (9a) by $R_{\text {cons-half, the }}$ constriction resistance of a semi-infinite half space (Equation 2):

$$
F=\left(4 k_{s u b} a\right) \frac{T_{z=L}+L\left[\frac{d T}{d z}\right]_{z=L}-T_{z=0}}{q}
$$




\section{RESULTS AND DISCUSSION}

The model was validated by comparing the values of the constriction alleviation factor $F$ for a semi-infinite cylinder with no frustum (Figure 4) obtained using the present model to those from the expression of Cooper et al. [10]. A comparison of the results is shown in Table 1. The two sets of values agree to within $2 \%$, confirming the validity of the present modeling approach. The problem of a semi-infinite cylinder terminating in the frustum of a cone is considered in the rest of this discussion.

The results for the constriction alleviation factor obtained from the present model are compared to the results of Olsen et al. [4] for conductivity ratio $k_{g a s} k_{s u b}=1.54 \times 10^{-4}$ in Figure 9 . The two models deviate significantly for small $a / b$ ratios, but the difference decreases as $a / b$ increases. The difference between the two models is also smaller when the contact angle is larger. The non-graded structured mesh used by Olsen et al. allows for only a few grid points to be included close to the contact spot and in the cone frustum. The large temperature gradients near the contact spot could thus not be fully resolved in their model, especially for small $a / b$ ratios. Using an unstructured mesh, the discretization in this region is much better accomplished without incurring significant increases in processing time, while obtaining much better accuracy even for small contact angles and $a / b$ values.

Numerical predictions of constriction resistance for various cylinder geometries and conductivity ratios are presented in Figures 10,11 and 12, in which the effects of the governing parameters, $a / b, \theta$ and $k_{g a s} / k_{s u b}$, on the constriction resistance are brought out. The constriction resistance $R_{\text {cons }}$ is plotted as a function of contact angle for extreme values of the $a / b$ and $k_{g a s} / k_{\text {sub }}$ ratios considered in this study. A constant value of $b=1 \mathrm{~m}$ was used for all the calculations. The constriction resistance increases monotonically with contact angle. This is because the heat 
flow through the gas gap provides alleviation in the constriction resistance, and as the contact angle increases, there is greater resistance to heat conduction across the gas gap, and hence less alleviation. Therefore the constriction resistance tends to increase as the contact angle increases. However, as $a / b$ increases the contact angle appears to have a decreasingly significant effect on the constriction resistance, especially when the conductivity of the substrate is high. As $a / b$ or $k_{s u b}$ increase, the fraction of the heat flowing through the gas gap relative to the total heat passing through the interface decreases significantly, and the changes to the geometry of the gas gap have a smaller effect.

The constriction resistance is plotted as a function of the conductivity ratio $k_{\text {gas }} k_{\text {sub }}$ for the extreme values of $a / b$ and $\theta$ in Figure 11. It is emphasized that these results are obtained for a fixed gas conductivity of $0.0242 \mathrm{~W} / \mathrm{mK}$ (air) with the substrate conductivity being varied from $15 \mathrm{~W} / \mathrm{mK}$ (stainless steel) to $415 \mathrm{~W} / \mathrm{mK}$ (silver) to obtain different values for the $k_{\text {gas }} / k_{\text {sub }}$ ratio. Therefore an increasing value for this ratio indicates a decreasing $k_{\text {sub }}$ and an increasing constriction resistance, as expected. As the conductivity of the substrate increases, the percentage of heat that is conducted through the gas gap relative to the total heat flow through the interface decreases. This supports the observation above that the contact angle has a smaller effect on the constriction resistance when the conductivity of the substrate is high. It may be noted that even when $a / b$ increases to 0.1 , the conductivity of the substrate still plays an important role in the constriction resistance. It was observed that if the conductivity of the substrate is held constant instead, and the conductivity of the gas varied, the constriction resistance shows a trend opposite to that in Figure 11; in that case, the constriction resistance increases with decreasing conductivity ratio. However, this does not represent a limitation in the modeling approach, since the constriction alleviation factor $F$ is a function only of the ratio of 
the two conductivities and is independent of the actual conductivities of the gas or substrate. As $F$ is a ratio of two resistances (Equation 3), it depends only on the fraction of the total heat flux that passes through the gas gap.

The variation of constriction resistance with $a / b$ is shown in Figure 12, again for the extreme values of $\theta$ and $k_{g a s} / k_{s u b}$ considered. As might be expected, the constriction resistance decreases as $a / b$ increases. It is especially apparent in this graph that as $a / b$ increases to 0.1 , the contact angle has little effect on the constriction resistance when the conductivity of the substrate is large. It may thus be inferred that for $a / b$ values of approximately 0.1 or greater, and for large substrate conductivities, the constriction resistance is dependent mainly on $a / b$. The conductivity of the gas and the contact angle have a very small effect, as reflected in the Cooper et al. [10] model of constriction resistance.

Using a nonlinear least-squares regression [15], the 3360 constriction resistance values computed in this work as a function of contact angle, $a / b$, and conductivity ratio were determined to be correlated according to the following expression:

$$
R_{\text {cons-cyl }}=\frac{1.608}{4 k_{\text {sub }} a}\left\{\left(1-\frac{a}{b}\right)^{\left[2.565(\kappa)^{0.141} \theta^{0.272}\right]}\right\}\left\{\theta^{\left[0.244(1-a / b)^{2.792}(\kappa)^{-0.612}\right]}\right\}\left\{\kappa^{\left[0.677(1-a / b)^{82.1} \theta^{-0.433}\right]}\right\}
$$

The conductivity ratio used in this correlation is given by $\kappa=k_{\text {sub }} /\left(k_{\text {sub }}+1000 k_{\text {gas }}\right)$. The effect of gas conductivity is captured using the parameter $\kappa$ in preference to the ratio $k_{\text {gas }} / k_{\text {sub }}$. If $k_{\text {gas }} k_{\text {sub }}$ were used as one of the factors in the correlation for $R_{\text {cons-cyl, }}$, the constriction resistance would take a non-physical value of zero for the case of a vacuum (i.e., for $k_{\text {gas }}=0$ ). Because $k_{\text {sub }}$ is in general 3-4 orders of magnitude higher than $k_{\text {gas }}$, the factor of 1000 ensures that changes in $k_{\text {gas }}$ are appropriately reflected in $\kappa$. The constriction alleviation factor $F$ for a semi-infinite cylinder terminating in the frustum of a cone may be obtained from this expression using the 
definition in Equation (3). This correlating equation is expected to be valid for the following ranges of parameter values: $0.01<a / b<0.1 ; 5.83 \times 10^{-5}<k_{\text {gas }} / k_{\text {sub }}<1.61 \times 10^{-3}$; and $0.0175<\theta$ $<0.628$. In Equation (10), the exponent for the $[1-(\mathrm{a} / \mathrm{b})]$ term ranges from 0.745 to 2.423 , as compared to an exponent of 1.5 in the expression of Cooper et al. (Equation 4). Also, the exponent for $\theta$ ranges from 0.188 to 0.427 , while the exponent for $\kappa$ ranges from $1.45 \times 10^{-4}$ to 1.712 .

The correlation for constriction resistance has an additional term $\left(\begin{array}{lll}k_{s u b} & a\end{array}\right)$ in the denominator. This is because the constriction resistance, in addition to depending on $a / b, \theta$ and $\kappa$, is also inversely proportional to the absolute values of the size ( $a$ and $b$ ) and the conductivities ( $k_{\text {gas }}$ and $\left.k_{\text {sub }}\right)$ of the domain. Moreover, the term $1 /\left(4 k_{\text {sub }} a\right)$ is the constriction resistance of a half-space of thermal conductivity $k_{s u b}$ with an opening of radius $a$ (Equation 2). This results in an elegant expression for $F$, which can be considered as a modification to the formula of Cooper et al. [10] (Equation 4), with extra terms included to account for the presence of the conical section and the conductive gas gap, which were not present in the geometry considered by Cooper et al.

The average deviation for all the data points from the values predicted by the correlation was $4.14 \%$ with a standard deviation of $17.3 \%$, while the maximum deviation of $32.84 \%$ occurred for $a / b=0.03, \theta=0.0175$, and $k_{\text {gas }} / k_{\text {sub }}=0.00161$ (stainless steel). The coefficient of determination, which indicates the amount of variability in the data captured by the correlation, is 0.999. The average deviation for the data points from Cooper's formula, on the other hand, was $25.64 \%$. The numerical values from the analysis are compared to the predicted values from the correlation for several different cases in Figure 13. The correlation is seen to successfully represent the results, with a larger error incurred when $a / b$ is small. The $F$ values from the 
numerical analysis are compared to the values predicted by the correlation in Figure 14, which shows that the correlation provides a good fit to the results. The constriction alleviation factor is seen to be somewhat underestimated by Equation (10) for several cases with $\mathrm{F}$ values ranging from $0.7-0.9$.

The variation of the constriction alleviation factor with $a / b$ is shown for different values of $k_{\text {gas }} / k_{s u b}$ and $\theta$ in Figure 15. The most noticeable point in the figure is that, when the substrate conductivity and the contact angle are small, $F$ is not a monotonically decreasing function of $a / b$. It may be expected that as the contact radius increases there would be greater alleviation of the constriction resistance and $F$ would decrease. However, $F$ represents the fraction of constriction resistance for the geometry considered here $\left(R_{\text {cons }}\right)$ as compared to the constriction resistance of a semi-infinite half space $\left(R_{\text {cons-half }}\right)$. While $R_{\text {cons-half }}$ decreases with increasing contact radius, $a$, it appears to do so at a faster rate than $R_{\text {cons }}$ resulting in an increase in $F$ with increasing $a$ (at small $a / b)$. This is not surprising because for the present geometry as $a / b$ increases, although there is a greater alleviation in the constriction resistance through the contact spot, there is also a concurrent decrease in alleviation through the gas gap. On the other hand, there is no such

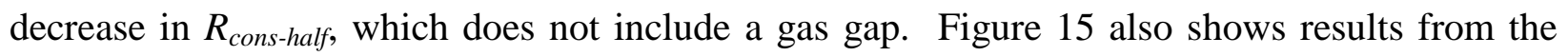
Cooper et al. [10] expression for constriction resistance. When the conductivity of the substrate is high and the angle $\theta$ is small the formula from Cooper et al. [10] is able to predict the constriction alleviation factor with good accuracy. However, there can be substantial deviation when the cone angle is large or when the conductivity of the substrate is low, since the significant alleviation due to the gas gap is not considered in [10]. 


\section{CONCLUSIONS}

A model for the constriction resistance which contributes to the thermal contact resistance between rough metallic surfaces was developed by numerically analyzing a semiinfinite cylinder terminating in the frustum of a cone. The effects of the ratio of contact radius to cylinder radius, contact angle, and ratio of gas gap conductivity to substrate conductivity on the constriction resistance are discussed. A comprehensive correlation for the constriction resistance as a function of these parameters is also presented. Since the ranges for the parameter values were obtained from information from surface scans of materials ranging from stainless steel to copper which were ground and bead-blasted to obtain several centerline average surface roughnesses ranging from 1 to $15 \mu \mathrm{m}$, most engineering surfaces are well represented by the correlation. In ongoing work, the effect of the presence of a coating on the substrate material is being examined to further expand the range of validity of the correlating formulas for constriction resistance.

\section{Acknowledgement}

The authors acknowledge the financial support of members of the Cooling Technologies Research Center (http://widget.ecn.purdue.edu/ CTRC), a National Science Foundation Industry/University Cooperative Research Center at Purdue University. 


\section{References}

[1] Yovanovich, M.M. and Antonetti, V.W., “Application of Thermal Contact Resistance Theory to Electronic Packages," Advances in Thermal Modeling of Electronic Components, Vol. 1, 1988, pp. 79-128.

[2]Madhusudana, C.V., Thermal Contact Conductance, Springer-Verlag, New York, 1996.

[3] Bowden, F.P., and Tabor, D., The Friction and Lubrication of Solids, Oxford University Press, London, 1950, pp. 20-32.

[4] Olsen, E.L., Garimella, S.V., and Madhusudana, C.V., "Modeling of Constriction Resistance in Coated Joints," AIAA Journal of Thermophysics and Heat Transfer, Vol. 16, 2002, pp. 207-216.

[5] Carslaw, H.S., and Jaeger, J.C., Conduction of Heat in Solids, 2nd edition, Clarendon Press, Oxford, 1959, pp. 214-217.

[6] Mohs, W.F., Madhusudana, C.V. and Garimella, S.V., "Constriction Resistance in Coated Joints," Proceedings of NHTC'00, August 20-22, 2000, pp.1-7.

[7] Mikic, B.B., and Rohsenow, W.M., 1966, “Thermal Contact Resistance,” Mechanical Engineering Department Report No. DSR 74542-41, MIT, Cambridge, MA.

[8] Gibson, R.D., "The Contact Resistance for a Semi-Infinite Cylinder in a Vacuum,” Applied Energy, Vol. 2, 1976, pp. 57-65.

[9] Negus, K.J., Vanoverbeke, C.A., and Yovanovich, M.M., "Thermal Constriction Resistance with Variable Conductivity near the Contact Surface," Fundamentals of Conduction and Recent Developments in Contact Resistance, ASME HTD-Vol. 69, 1987, pp. 91-98.

[10] Cooper, M.G., Mikic, B.B., and Yovanovich M.M., “Thermal Contact Conductance,” Int. J. Heat Mass Transfer, Vol. 12, 1969, pp. 279-300.

[11] Madhusudana, C.V., "Heat Flow Through Conical Constrictions in Vacuum and in Conducting Media," AIAA Paper 79-14071, New York, 1979. 
[12] Singhal, V., and Garimella, S.V. "Prediction of Thermal Contact Conductance by Surface Deformation Analysis," in Proceedings of International Mechanical Engineering Congress and Exposition, New York, IMECE2001/HTD-24376, pp. 1-8, November 2001.

[13] Fluent Inc., "Gambit 2.0 User’s Guide,” Lebanon, NH, Fluent Inc, 2002.

[14] Fluent Inc., "Fluent 6.0 User's Guide,” Lebanon, NH, Fluent Inc, 2000.

[15] Chapra, S.C., and Canale R.P., Numerical Methods for Engineers, $3^{\text {rd }}$ edition, McGraw Hill, 1998, pp. 423-560. 
Table 1. Comparison of results for the semi-infinite cylinder model.

\begin{tabular}{|c|c|c|}
\hline \multirow{2}{*}{$a / b$} & \multicolumn{2}{|c|}{$F$} \\
\cline { 2 - 3 } & Cooper et al. [10] & Present \\
\hline 0.01 & 0.9850 & 0.9796 \\
\hline 0.1 & 0.8538 & 0.8630 \\
\hline 0.2 & 0.7155 & 0.7296 \\
\hline 0.4 & 0.4648 & 0.4624 \\
\hline
\end{tabular}

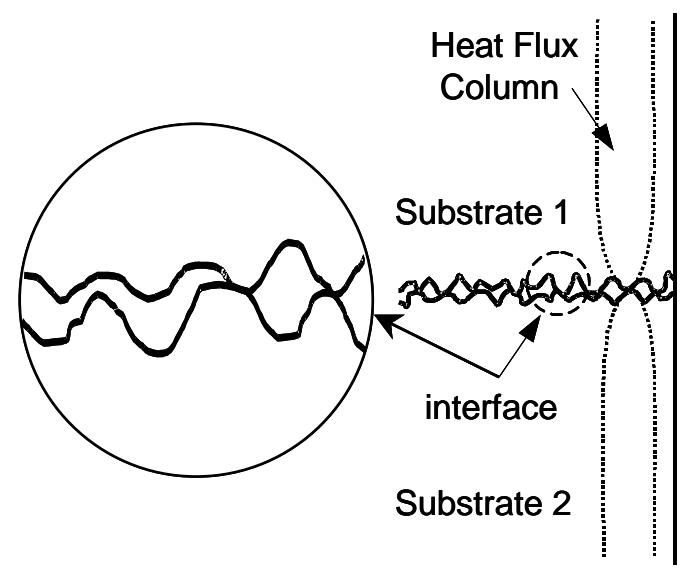

Figure 1. Constriction and spreading of heat flow lines at a rough interface.

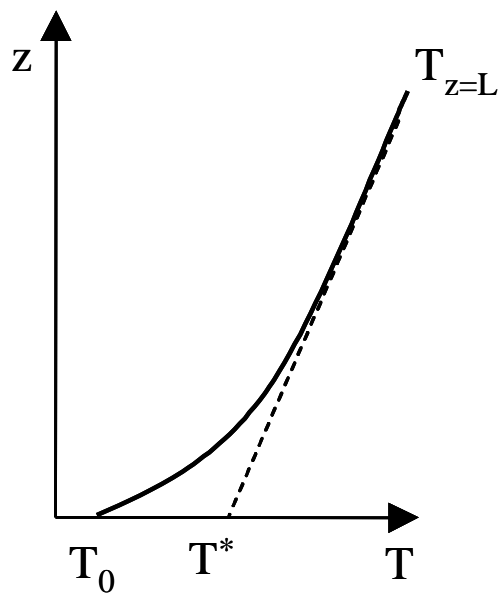

Figure 2. Temperature profile at a contact spot. 


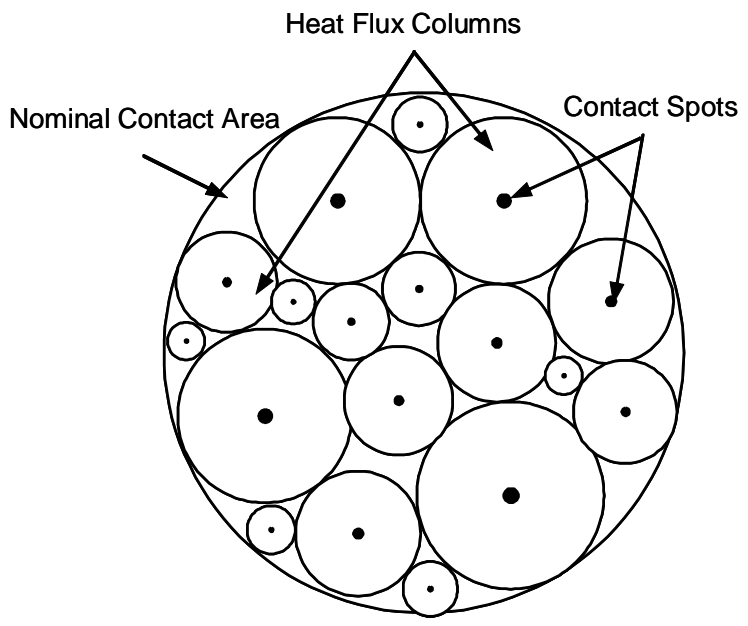

Figure 3. Constriction resistance at a real interface may be modeled as several semi-infinite cylindrical heat flow columns feeding contact spots at the interface.

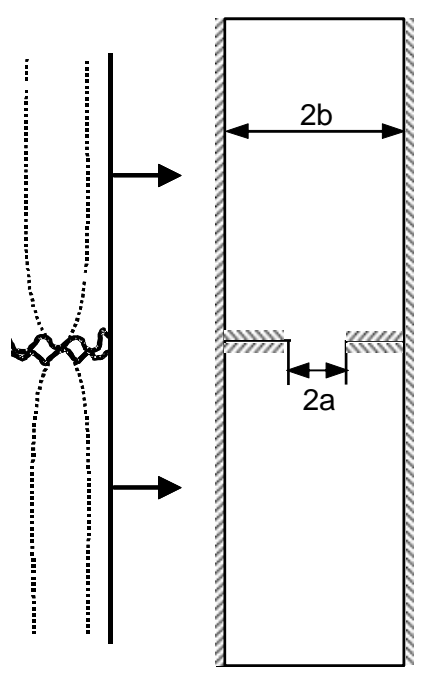

Figure 4. Semi-infinite cylinder constriction resistance model. 


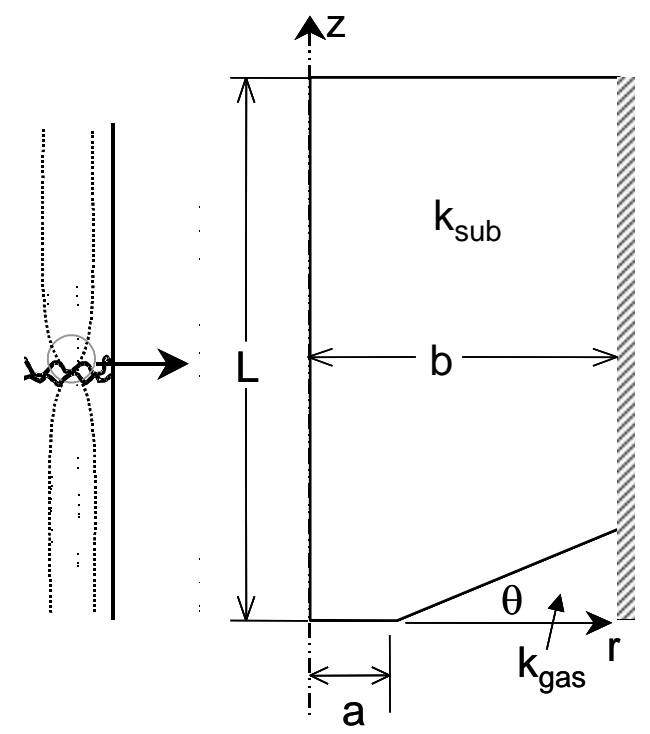

Figure 5. Semi-infinite cylinder terminating in the frustum of a cone.

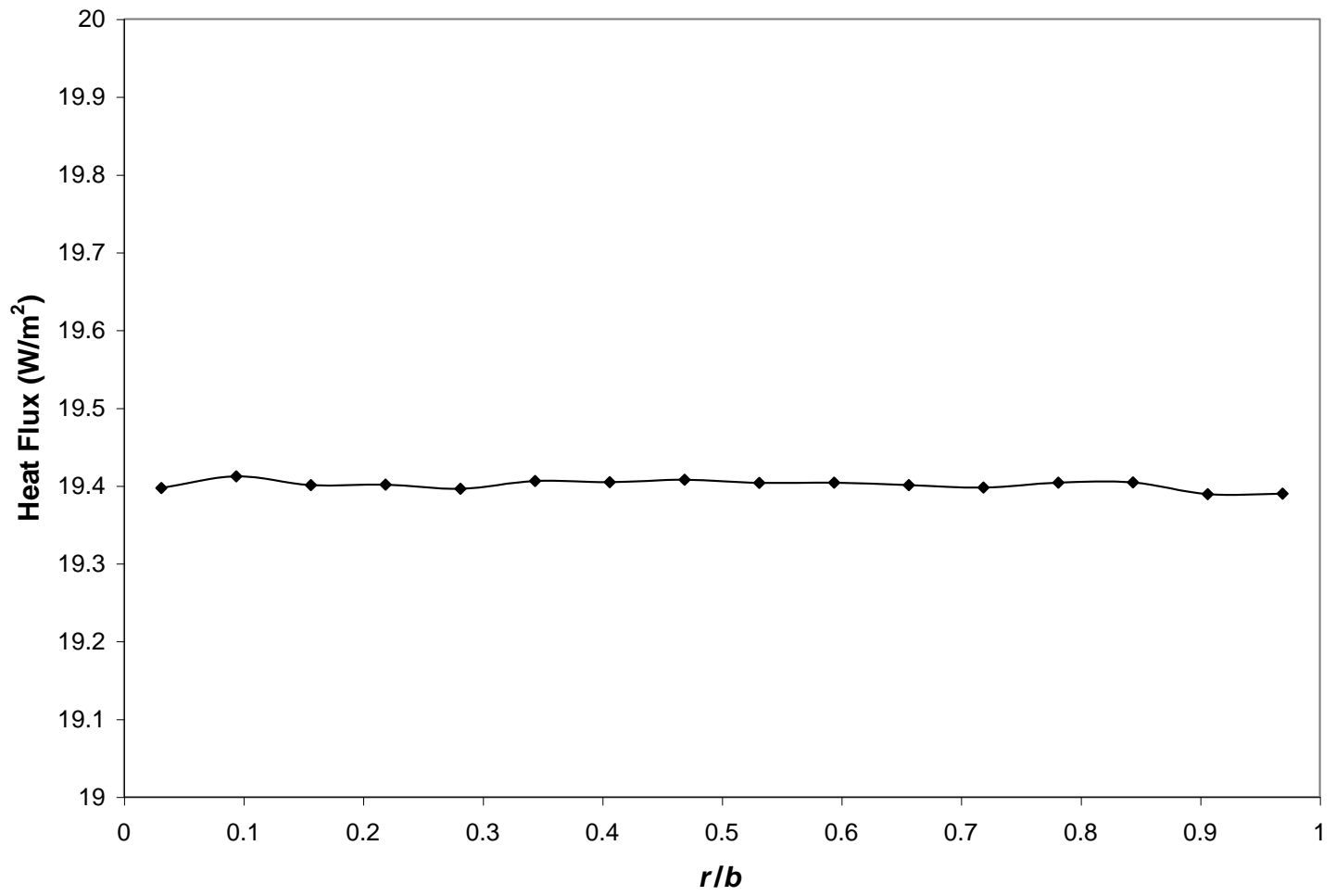

Figure 6. Heat flux across the top of the domain $(\mathrm{z}=\mathrm{L})$ for one of the cases where the constriction resistance is the greatest; flux is seen to be uniformly distributed far from the constriction. 


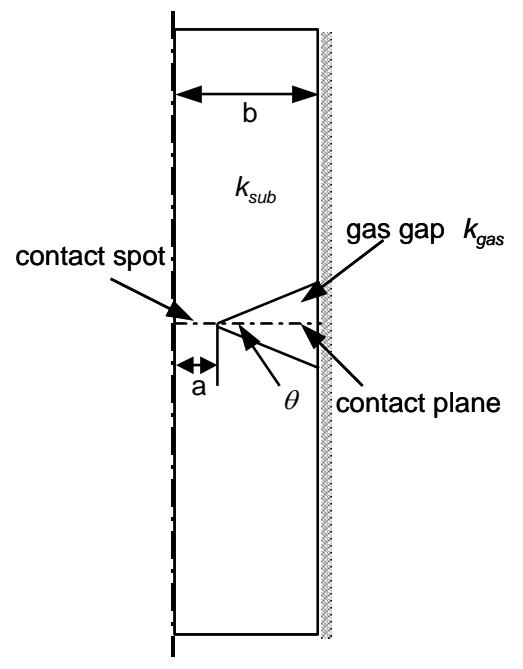

Figure 7. Domain used in determining the validity of the constant temperature assumption at the contact interface.

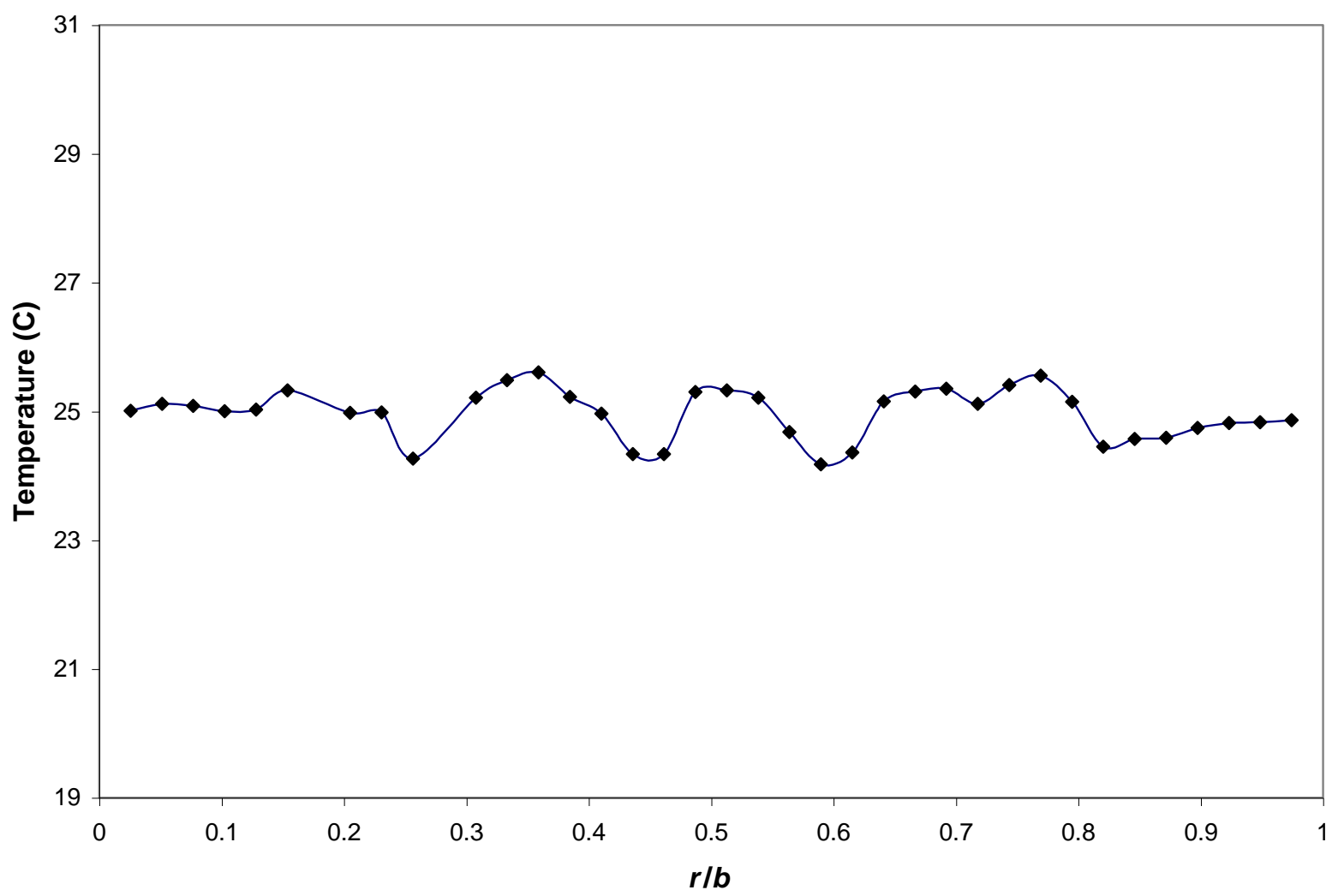

Figure 8. Temperature profile at the interface plane for the domain shown in Figure 7 with $\theta=12, a / b=0.1, k_{\text {gas }} / k_{\text {sub }}=1.2 \times 10^{-4}$. 


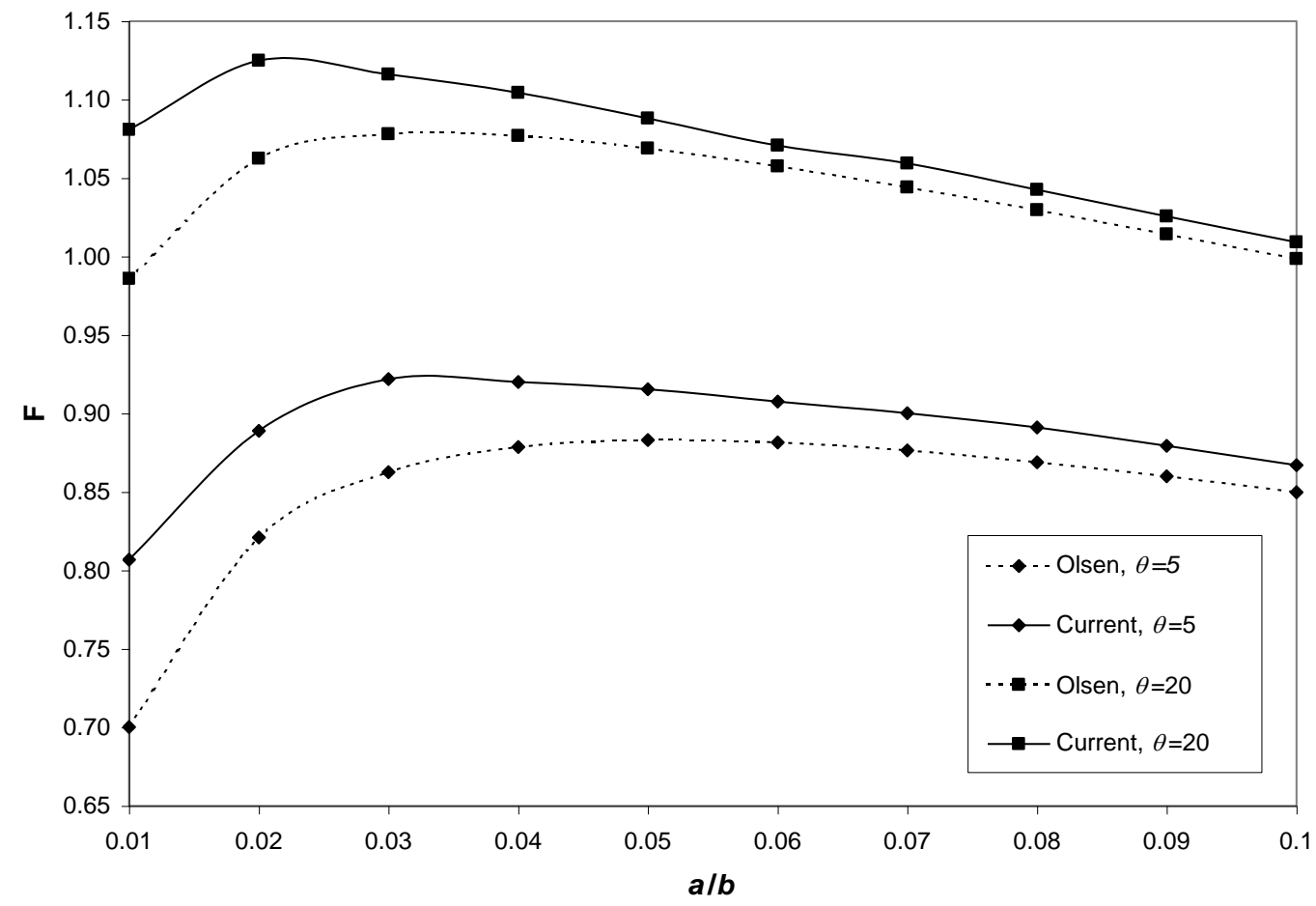

Figure 9. Comparison of constriction alleviation factors obtained from present work (unstructured mesh) to those from Olsen et al. [4] (structured mesh). 


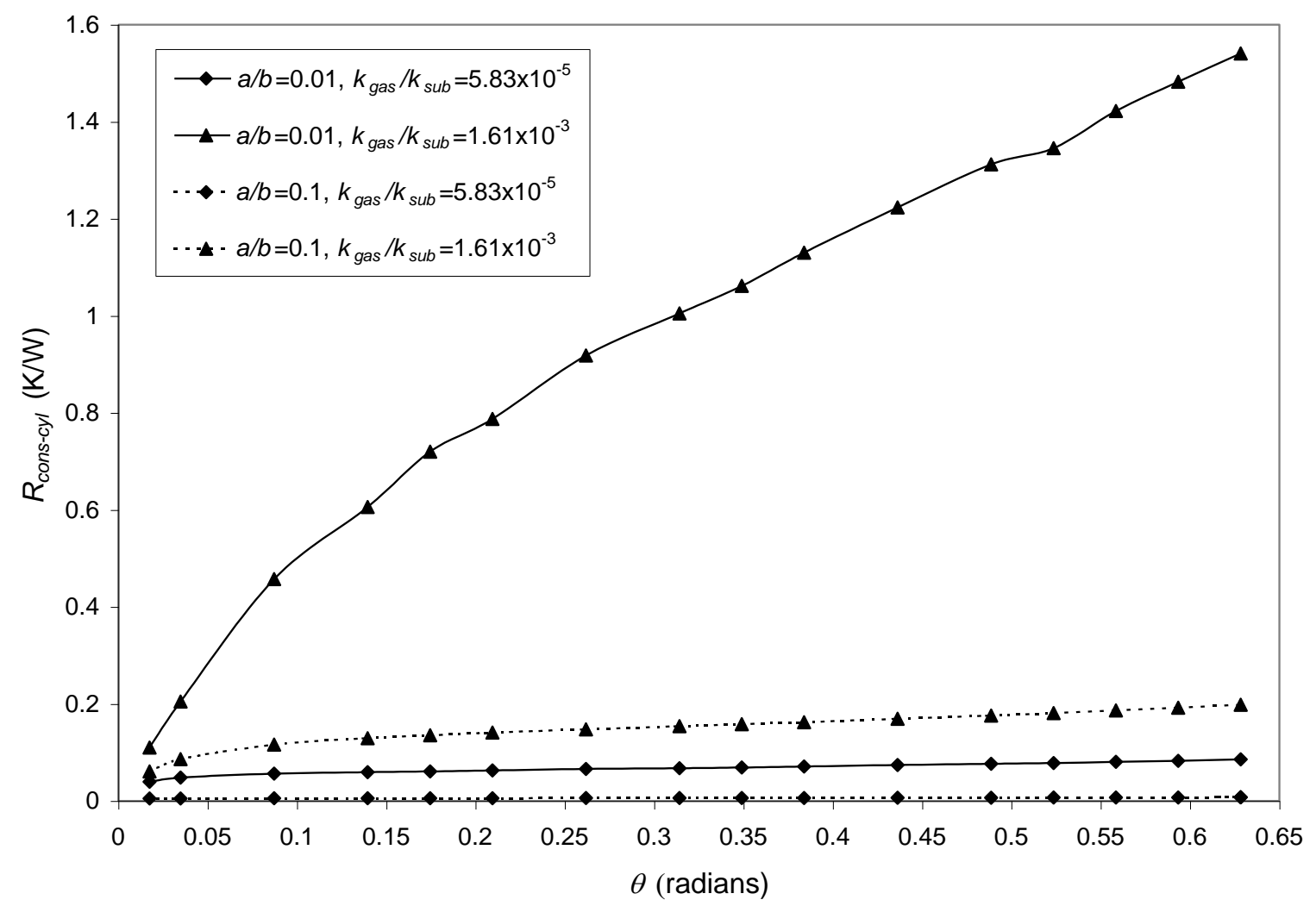

Figure 10. Effect of contact angle on constriction resistance for various contact spot radii and conductivity ratios. 


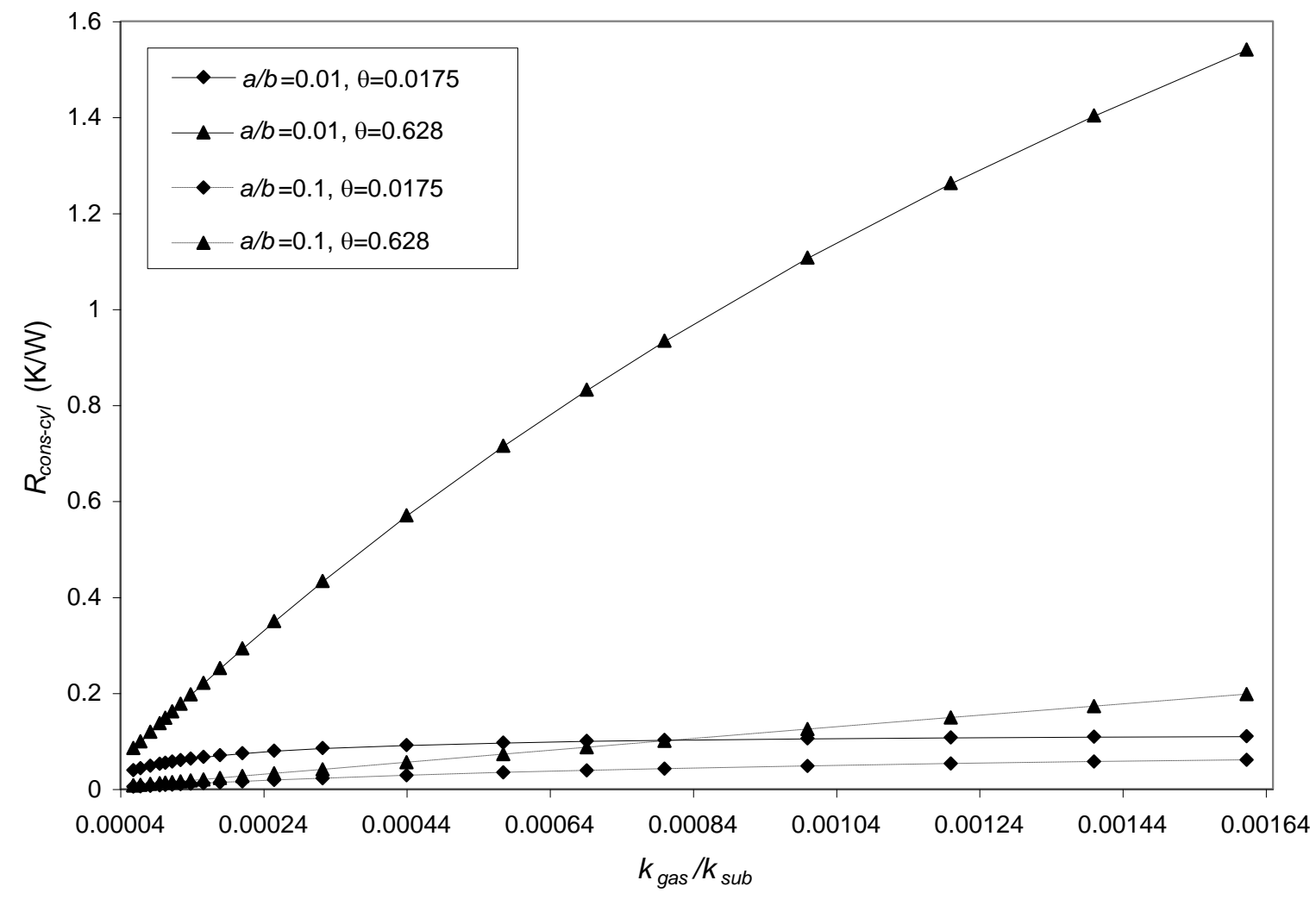

Figure 11. Effect of substrate conductivity on the constriction resistance for various cylinder geometries in an environment of air. 


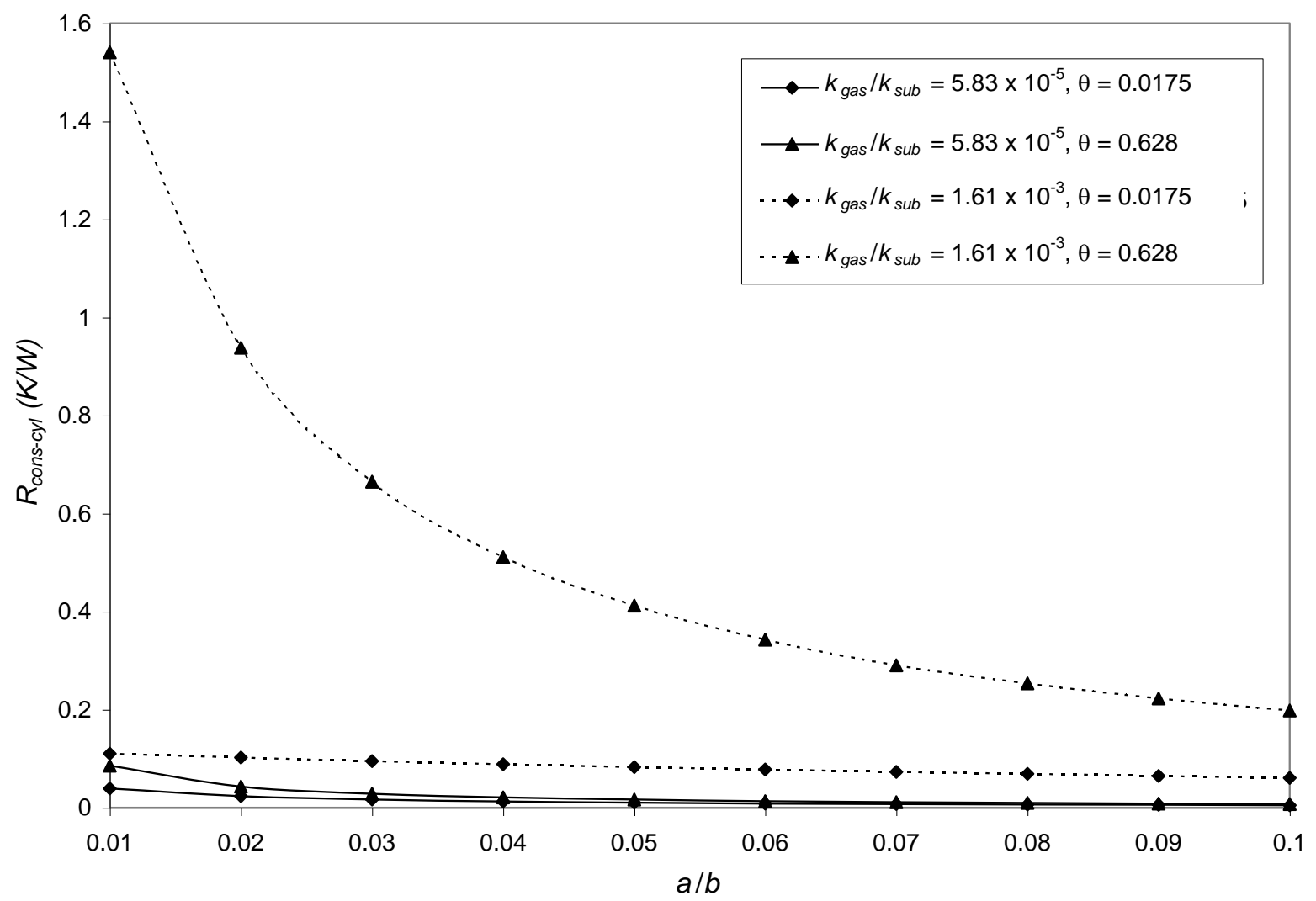

Figure 12. Effect of contact spot radius on the constriction resistance for various contact angles and conductivity ratios. 


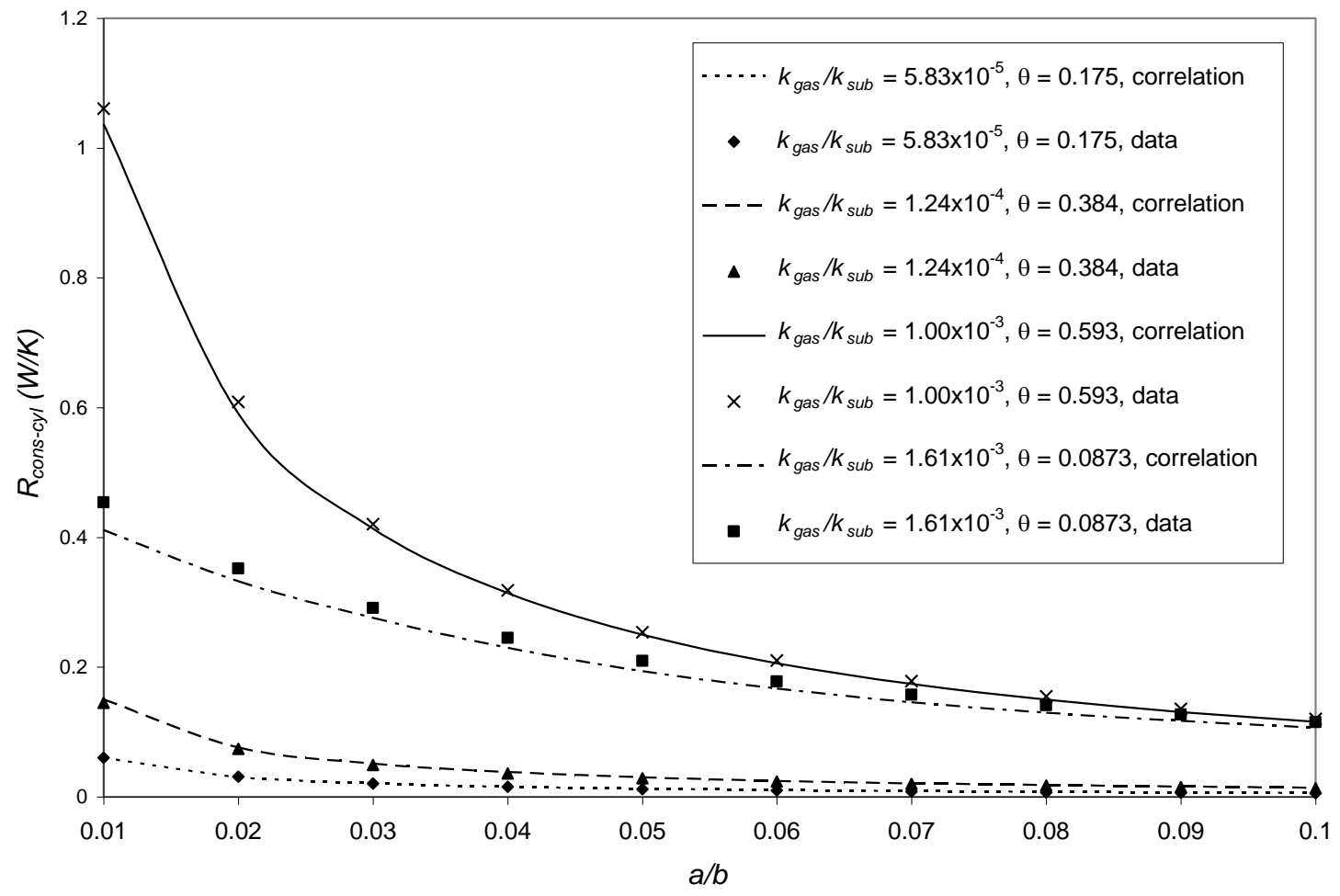

Figure 13. Comparison of predictions from the correlation to numerically computed values of constriction resistance. 


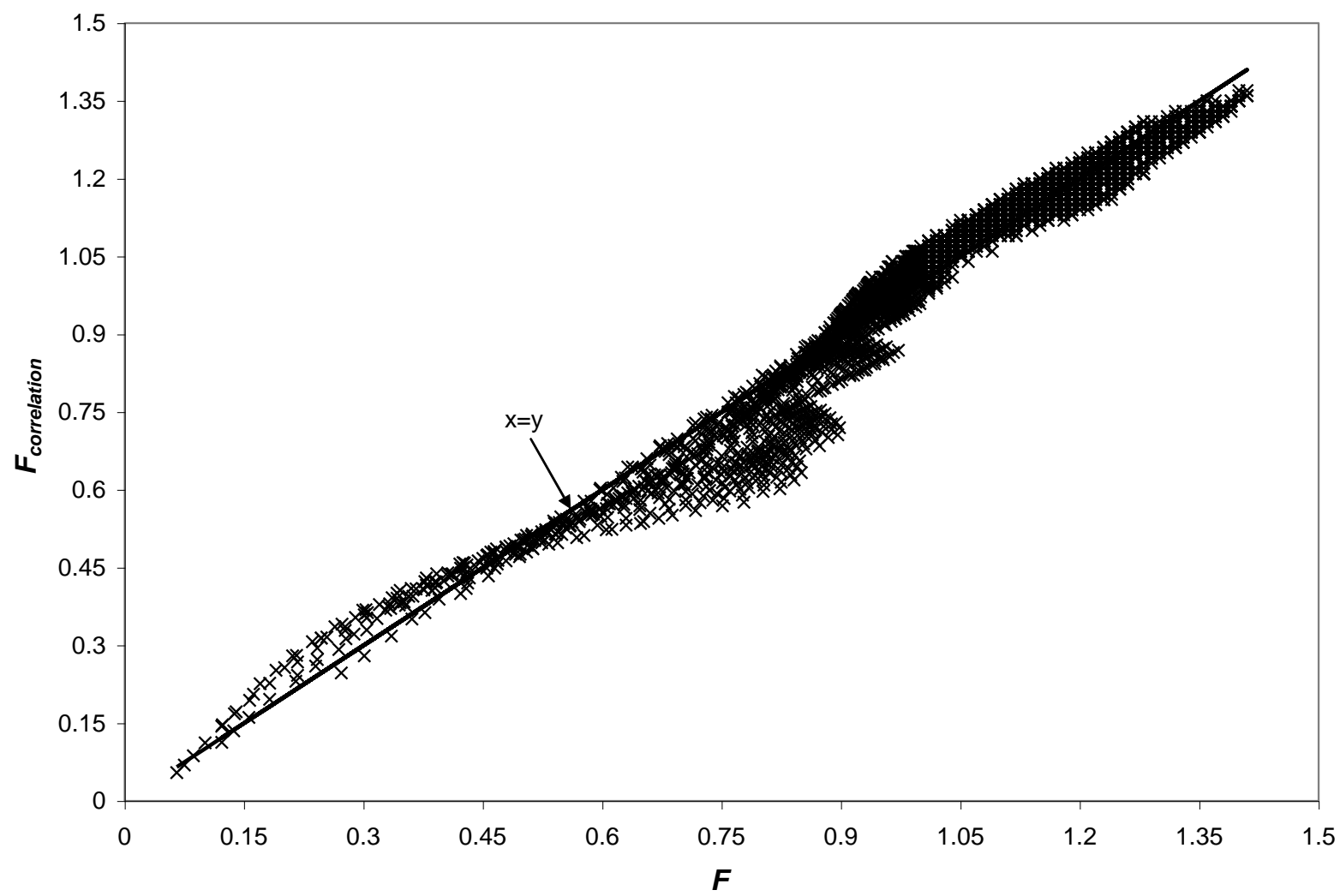

Figure 14. Comparison of constriction resistance factors from the correlation to values directly computed from the numerical analysis. 


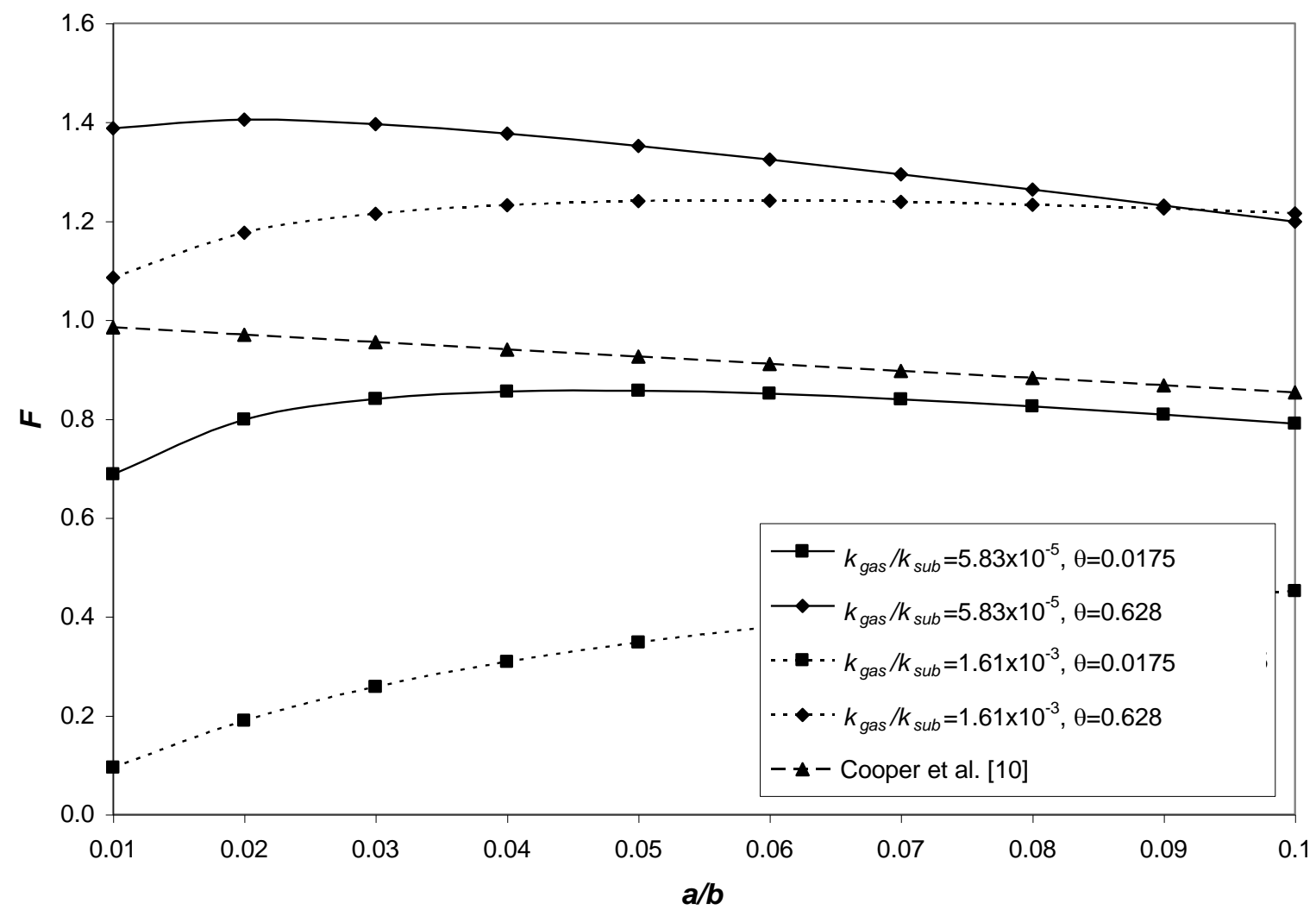

Figure 15. Variation of constriction alleviation factor with $a / b$. 